UDC 530.19

\title{
ON THE STRUCTURE OF COHOMOLOGICAL MODELS OF ELECTRODYNAMICS AND GENERAL RELATIVITY
}

\author{
Arkhipov V.V. ${ }^{1}$, Aringazin A.K. ${ }^{2}$, Kudussov A.S. ${ }^{3}$ \\ 1Moscow Institute of Physics and Technology, Moscow, 141701, Russia, arkhipov.vv@mipt.ru \\ 2 L.N. Gumilyov Eurasian National University, Nur-Sultan, Kazakhstan, aringazin@gmail.com \\ ${ }^{3}$ E.A. Buketov Karaganda University, Karaganda, Kazakhstan, akudusov@mail.ru
}

\begin{abstract}
In the present paper, we take case of a complex scalar field on a Riemannian manifold and study differential geometry and cohomological way to construct field theory Lagrangians. The total Lagrangian of the model is proposed as 4-form on Riemannian manifold. To this end, we use inner product of differential (p, q)-forms and Hodge star operators. It is shown that actions, including that for gravity, can be represented in quadratic forms of fields of matter and basic tetrad fields. Our study is limited to the case of the Levi-Civita metric. We stress some features arisen within the approach regarding nil potency property. Within the model, Klein-Gordon, Maxwell and general relativity actions have been reproduced.
\end{abstract}

Keywords: cohomological theory, exterior calculus, differential forms, field theory, Riemannian manifold.

\section{Introduction}

A cohomological approach is used to describe field theory models as a powerful instrument to reveal hidden symmetries and especially topological properties. The use of the theory of differential forms and external calculus is a feature of Einstein-Cartan's theory [1,2], teleparallel gravity [3] and field theories on symplectic manifolds [4]. We note that the action for gravitational field in general relativity introduced as a 4-volume integral of the scalar curvature of the field, or other scalars are built from metrics and connections (see, for example a review work [5]). The reason for such choice is to keep invariance and follow minimal model set up. Similar approach is used in the gauge theory of gravity, where the covariant derivative is introduced by analogy with the theories of physical fields, but the action of the field itself is postulated in the standard form used in general relativity; see, for example, one of the first work [6].

In our approach, we build the action based on the well-known inner product of differential forms,

$$
(\xi, \varphi)=\int \xi \wedge * \varphi_{z}
$$

where $*$ is the Hodge star operator. The concept of external differential forms is generalized to the objects defined simultaneously on the tangent and cotangent bundle and written in the antisymmetric basis,

$$
\omega=\frac{1}{p !} \frac{1}{q !} \omega_{\alpha_{1} \cdots}^{\beta_{1} \cdots} \beta_{q} \beta_{q} d x^{a_{1}} \wedge \ldots \wedge d x^{\alpha_{p}} \wedge \partial_{\beta_{1}} \wedge \ldots \wedge \partial_{\beta_{q}}
$$

The objects are well-known and called $(p, q)$-forms. We introducing these and use in such a way that the action can be constructed in a cohomological approach.

We use the following concepts and notations. Let $x^{\mu}$ be coordinates in a certain area of the Riemannian manifold $\mathrm{R}^{1,3}$. We naturally assume that a small neighborhood of each point $\mathrm{R}^{1,3}$ is isomorphic to Minkowski space. That is, at each point $\mathrm{R}^{1,3}$ a local coordinate system $x^{a}(a=0,1,2,3)$ can be chosen, in which the metric takes the simple form $\eta_{a b}=\operatorname{diag}(1,-1,-1,-1)$.

Here below, we use Latin letter indices for local coordinate system and $x^{a}$ Greek letter indices for the global system $x^{\mu}$ on $\mathrm{R}^{1,3}$. Thus at each point on $\mathrm{R}^{1,3}$ the following elements are defined: the elementary interval $d s=\sqrt{\eta_{a b} d x^{a} d x^{b}}$, the elementary oriented area $d x^{a} \wedge d x^{b}$, volume $d x^{a} \wedge d x^{b} \wedge d x^{c}$, and 
4-volume form $\Omega=\frac{1}{4 !} \epsilon_{a b c d} d x^{a} \wedge d x^{b} \wedge d x^{c} \wedge d x^{d}$, where $\epsilon_{a b c d}$ is totally antisymmetric tensor; $\epsilon_{0123}=1$.

The relation of the differentials of the local coordinate system $d x^{a}$ with the differentials $d x^{\mu}$ at a given point is determined by the coefficients $h_{\mu}^{a}$ and $h_{a}^{\mu}\left(h_{\mu}^{a} h_{b}^{\mu}=\delta_{b}^{a}, h_{\mu}^{a} h_{a}^{v}=\delta_{\mu}^{v}\right)$ :

$$
d x^{a}=h_{\mu}^{a} d x^{\mu}, \quad d x^{\mu}=h_{a}^{\mu} d x^{a} .
$$

If we define a basis $d x^{\alpha}$ independently at each point of the space $\mathrm{R}^{1,3}$ then the coefficients $h_{\mu}^{\alpha}$ and $h_{\alpha}^{\mu}$ turn into functions of the system $h_{\mu}^{a}\left(x^{\mu}\right), h_{a}^{\mu}\left(x^{\mu}\right)$. Since $d x^{a}$ is independent at different points in space, they can be considered as a tetrad of basis fields $h^{a}\left(x^{\mu}\right)$. These are not necessarily holonomic ones [3].

The space-time metric on $\mathrm{R}^{1,3}$ in the holonomic basis $d x^{\mu}$ is definedin the standard way: $g_{\mu v}=\eta_{a b} h_{\mu}^{a} h_{v}^{b}$.

In the following, along with the notation $d x^{\mu}$ and $d x^{a}$ for the anti-symmetric basis with multiplication denoted by $\wedge$, we will use notation $c^{\mu}$ and $c^{a}$, assuming the antisymmetric property $c^{\mu} c^{v}=-c^{v} c^{\mu}$.

On $\mathrm{TR}^{1,3}$, an antisymmetric basis dual to $c^{\mu}$ can be introduced, which we denote as $\bar{c}_{\mu}$. In this basis, contravariant antisymmetric tensors called here as $q$-forms, dual to the usual antisymmetric differential $p$ forms, can be expanded. In particular, vector space can beun derstood due to isomorphism as the space of contravariant 1 -forms $\xi=\xi^{\mu} \bar{c}_{\mu}$. Generally, $c^{a}\left(x^{\mu}\right)$ can be treated not only as the differentials of $d x^{a}$. For example, one could assign it Fadeev-Popov's ghost fields meaning; see [7] for details. In this set up, the fields $c^{\mu}$ and $\bar{c}_{\mu}$ acquireghost numbers

$$
\text { ghost\# }\left(c^{\mu}\right)=1, \text { ghost } \#\left(\bar{c}_{\mu}\right)=-1
$$

and the total ghost number should be conserved.

The basis for contravariant vectors is usually denoted as $\partial / \partial x^{\mu}$, as a dual to the differentials of $d x^{\mu}$. The choice of such a notation is determined by the corresponding law of the gradient transformationof the function $\partial_{\mu} f\left(x^{a}\right)$. Geometrically, the main this is their transformation rules. Taking into account the possibility of a scalar product between basic elements on $\mathrm{TR}^{1,3}$ and $\mathrm{T} * \mathrm{R}^{1,3}$, it is more convenient to associate with $\bar{c}_{\mu}$ the operator $\partial / \partial c^{\mu}$ rather than $\partial_{\mu}$. In the conjugate representation, $c^{\mu}$ can be associated with $\partial / \partial \bar{c}_{\mu}$. We stress that the $c^{a}$ introduced above are the same as $h^{a}$ but antisymmetric multiplication through the wedge $\wedge$ should be used.

\section{External Calculus on Riemannian Manifold}

Let $p$-forms be ordinary differential antisymmetric forms defined on the cotangent bundle $\mathrm{T} * \mathrm{R}^{1,3}$. They can be written in a form

$$
\omega=\frac{1}{p !} \omega_{\mu_{1} \cdots \mu_{p}} \mathrm{dx}^{\mu_{1}} \wedge \ldots \wedge \mathrm{dx} x^{\mu_{p}} \quad \text { or } \quad \omega=\frac{1}{p !} \omega_{\mu_{1} \ldots \mu_{p}} \mathrm{c}^{\mu_{1}} \ldots \mathrm{dc}^{\mu_{p}}
$$

Let $q$-forms be contravariant antisymmetric tensors of rank $q$ defined on a tangent bundle $\mathrm{TR}^{1,3}$. They have a form

$$
\chi=\frac{1}{q !} \chi^{\mu_{1} \cdots} \mu_{q} \partial_{\mu 1} \wedge \ldots \wedge \partial_{\mu q} \quad \quad \text { or } \quad \chi=\frac{1}{q !} \chi^{\mu_{1} \cdots \mu_{q}} \bar{c}_{\mu 1} \ldots \bar{c}_{\mu q}
$$

Let $(p, q)$-forms be antisymmetric tensors with $q$ contravariant and $p$ covariant indices. The tensors are antisymmetric with respect to the both sets of contravariant and covariant indices. Therefore, the $(p, q)$-forms defined simultaneously on the tangent $\mathrm{TR}^{1,3}$ and on the cotangent $\mathrm{T}^{*} \mathrm{R}^{1,3}$ bundles and can be written as 


$$
\eta=\frac{1}{p !} \frac{1}{q !} \eta_{v_{1} \cdots}^{\mu_{1} \cdots} \cdot{ }_{v_{p}}^{\mu_{q}} c^{v_{1}} \ldots c^{v_{p}} \bar{c}_{\mu 1} \ldots \bar{c}_{\mu q} .
$$

There is an isomorphism between the tangent and cotangent bundles realized by metric operators

$$
\widehat{g}=g_{\mu v} c^{\mu} \frac{\partial}{\partial \bar{c}_{v}}, \hat{g}^{-1}=g^{\mu v} \bar{c}_{\mu} \frac{\partial}{\partial c^{v}} .
$$

Let's define the generalizations of these operators $\widehat{G}$, which trans-form $(p, q)$-forms into $(q, p)$-forms using the metric tensor due to the rule

$$
\widehat{G}_{\eta}=\frac{1}{p !} \frac{1}{q !} \eta_{v_{1} \cdots}^{\mu_{1} \cdots} \bar{v}_{p}^{\mu_{q}} g_{\mu_{1} a_{1}} \ldots g_{\mu_{q} a_{q}} g^{v_{1} \beta_{1}} \ldots g^{v_{p} \beta_{p}} c^{a_{1}} \ldots c^{a_{q}} \bar{c}_{\beta_{1}} \ldots \bar{c}_{\beta_{p}} .
$$

A covariant exterior differential $d$ that converts $(p, q)$-forms to $(p+1, q)$-forms can be defined as $d=c^{\varpi} \nabla_{\varpi}$, or

$$
d=c^{\alpha} \partial_{\alpha}+c^{\alpha} \Gamma_{\alpha \beta}^{\gamma} \bar{c}_{y} \frac{\partial}{\partial \bar{\epsilon}_{\beta}} .
$$

In particular, when it is acting on $(0,1)$-form $\chi=\chi_{\tau} \bar{c}_{\tau}$ we have:

$$
d_{\chi}=\left(c^{\alpha} \partial_{\alpha}+c^{\alpha} \Gamma_{\alpha \beta}^{\gamma} \bar{c}_{y} \frac{\partial}{\partial \bar{c}_{\beta}}\right) \chi_{\tau} \bar{c}_{\tau}=c^{\alpha} \nabla_{\alpha} \chi^{\beta} \bar{c}_{\beta} .
$$

Here and after, by $\Gamma_{a \beta}^{\gamma}$ is meant the Levi-Civita connection, consistent with the metric

$$
\Gamma_{\alpha \beta}^{Y}=\frac{1}{2} g^{\kappa \lambda}\left(\partial_{\kappa} g_{\lambda \beta}+\partial_{\beta} g_{\lambda \alpha}-\partial_{\lambda} g_{\alpha \beta}\right) \text {. }
$$

Note that the nil potency property of the exterior differential $d^{2}=0$ is preserved on the space of $(p, 0)$ forms. However, on the space of $(0, q)$-forms this property is violated. In particular, for $\chi=\chi_{\tau} \bar{c}_{\tau}$ we have

$$
d^{2} \chi=\frac{1}{2} c^{\alpha} c^{\mu} \bar{c}_{\alpha} R_{\mathrm{v} \alpha \mu}^{\alpha} \chi^{\mathrm{v}}
$$

where $R_{\text {vap }}^{\alpha}$ is the Riemannian curvature tensor.

It can be shown that the dual to $d$ operator $\bar{d}=\bar{G} d \bar{G}$ that converts $(p, q)$-forms to $(p, q+1)$-forms can be represented as $\bar{d}=\bar{c}_{\kappa} g^{\alpha \beta} \nabla_{\beta}$, or

$$
\bar{d}=\bar{c}_{\alpha} g^{\alpha \beta}\left(\partial_{\beta}+\Gamma_{\lambda \beta}^{Y} \bar{c}_{Y} \frac{\partial}{\partial \bar{c}_{\lambda}}-\Gamma_{\lambda \beta}^{\gamma} c^{\lambda} \frac{\partial}{\partial \sigma^{\gamma}}\right) .
$$

Using the 4-form of volume $\Omega=\frac{1}{4 !} \sqrt{-g} \epsilon_{\mu_{1} \mu_{2} \mu_{8} \mu_{4}} c^{\mu_{1}} c^{\mu_{2}} c^{\mu_{g}} c^{\mu_{4}}$ and its dual, fully antisymmetric contravariant tensor $\bar{\Omega}=\frac{1}{4 !} \frac{1}{\sqrt{-g}} \epsilon^{\mu_{1} \mu_{2} \mu_{\mathrm{g}} \mu_{4}} \bar{c}_{\mu_{1}} \bar{c}_{\mu_{\Omega}} \bar{c}_{\mu_{\mathrm{g}}} \bar{c}_{\mu_{4}}$, we can define two types of the Hodge star operators: the operator $*$ which is transforming $(p, q)$-forms into $(4-p, q)$-forms in a standard way and the conjugate operator $*$ converting $(p, q)$-forms into $(p, 4-q)$-forms.

The Hodge star operators $*$ and $*$ allow us to construct the divergence operator $\hat{\delta}=* d *$, lowering the $p$-rank of the form by one $((p, q) \rightarrow(\mathrm{p}-1, \mathrm{q}))$, and its dual operator $\hat{\delta}=\star \bar{d} \star$ acting like $(p, q) \rightarrow(p, q-1)$. It can be shown that 


$$
\hat{\bar{\delta}}=\partial_{\mu} \frac{\partial}{\partial \bar{c}_{\mu}}+\frac{\partial}{\partial \bar{\epsilon}_{\alpha}} c^{\mathrm{v}} \Gamma_{\mathrm{v} \mu}^{\alpha} \frac{\partial}{\partial \bar{\epsilon}_{\mu}} .
$$

Note that the operator can be derived from (2) by formal replacing $c^{\alpha} \rightarrow \partial / \partial \bar{c}_{\alpha}$.

It is easy to check that commutators $[d, \hat{\delta}]$ and $[\bar{d}, \hat{\delta}]$ give the same Laplace operator (up to the isomorphism $\hat{G}$ ). We use the term 'commutator' both to commutators and anti-commutators assuming that the latter applies only for odd $(p+q)$ rank. We now see that methods of cohomology theory (see for example [8]) can be applied here to a full extent. However, it is easy to verify that the commutator of conjugate operators (2) and (3) gives the Ricci tensor when acts at the (0,1)-form:

$$
(d \hat{\bar{\delta}}+\hat{\bar{\delta}} d) \xi=-c^{a} R_{a \tau} \xi^{\tau}
$$

The inner product (1) allows to build entities like usual scalar product but it has a wider scope. In this paper, we are using the following formulas. For usual functions (0-forms) $f\left(x^{\mu}\right)$ and $f\left(g^{\mu}\right)$ we have

$$
(f, g)=\frac{1}{4 !} \int f g \epsilon_{\lambda a \beta y} \sqrt{-g} d x^{\lambda} \wedge d x^{a} \wedge d x^{\beta} \wedge d x^{\gamma}=\int f g \sqrt{-g} d \Omega \text {, }
$$

where $d \Omega=d x^{0} d x^{1} d x^{2} d x^{3}$ is an elementary 4-volume.

For 1-forms $\phi_{1}=\phi_{\mu} c^{\mu}$ and $\varphi_{1}=\varphi_{\mu} c^{\mu}$ we have

$$
\left(\phi_{1}, \varphi_{1}\right)=\int \phi_{\mu} \varphi_{v} g^{\mu v} \sqrt{-g} d \Omega
$$

For 2-forms $\omega_{2}=(1 / 2) \omega_{\mu v} c^{\mu} c^{v}$ and $\sigma_{2}=(1 / 2) \sigma_{\mu v} c^{\mu} c^{v}$ it follows

$$
\left(\omega_{2}, \sigma_{2}\right)=2 \int \omega_{\mu v} \sigma_{\mathrm{a} \beta} g^{a \lambda} g^{\beta p} \sqrt{-g} d \Omega
$$

\section{Scalar Field}

The complex scalar field $\phi\left(x^{\mu}\right)$ can be considered as taking values in 'inner' spaces attached to the points of $\mathrm{R}^{1,3}$, with a complex structure $\mathrm{C}^{1}$ so that $\phi=\phi^{1}+i \phi^{2}$, or Euclidean one $\mathrm{R}^{2}$ with metrics $\tilde{\eta}_{A B}=\operatorname{diag}(1,1)$. In the last case,

$$
\phi=\phi^{A}=\left(\phi^{1}, \phi^{2}\right) .
$$

The inner product of two complex scalar fields $\phi$ and $\varphi$ gives the invariant with respect to the action of transformation group $\mathrm{U}(1)$,

$$
\left(\varphi^{\dagger}, \phi\right)=\int \varphi^{\dagger} \Lambda * \phi
$$

where the Hermitian conjugation $\dagger$ is acting only on the internal space. In particular, in the complex representation $\dagger$ is reduced simply to the complex conjugation and in the matrix representation to transpose. As it follows from (5) and (6) the inner square of the complex scalar field $\phi=\phi\left(x^{\mu}\right)$ and its first differential are

$$
\left(\phi^{*}, \phi\right)=\int|\phi|^{2} \sqrt{|g|} d \Omega,
$$

and

$$
\left(d \phi^{*}, d \phi\right)=\int g^{\mu v} \partial_{\mu} \phi^{*} \partial_{v} \phi \sqrt{|g|} d \Omega .
$$


This allows us to represent the action of a complex scalar field,

$$
S_{\phi}=\frac{1}{2} \int\left(g^{\mu v} \partial_{\mu} \phi^{*} \partial_{v} \phi-m^{2} \phi^{*} \phi\right) \sqrt{|g|} d \Omega,
$$

in the following form:

$$
S_{\phi}=\frac{1}{2}\left(d \phi^{*}, d \phi\right)-\frac{m^{2}}{2}\left(\phi^{*}, \phi\right) .
$$

This action can be varied directly using properties of the inner product (see [9]) and one can get usual Klein-Gordon equation in the form $\left(\partial^{\mu} \partial_{\mu}-m^{2}\right) \phi=0$. To turn on an interaction with external electromagnetic field let's consider a basis on an 'inner' space $\mathrm{e}_{\mathrm{A}}(\mathrm{A}=1,2)$. Let's define an internal connection in standard way as

$$
\delta e_{A}=e_{\beta} \widetilde{\Gamma}_{A \mu}^{\beta} \delta x^{\mu},
$$

with imposed local U(1) symmetry we get

$$
\widetilde{\Gamma}_{A \mu}^{\beta}=S_{A}^{\beta} A_{\mu}\left(x^{\mu}\right),
$$

where $S_{A}^{\beta}=\left(\begin{array}{cc}0 & 1 \\ -1 & 0\end{array}\right)$ is a generator of $\mathrm{u}(1)$ algebra.

Thus, the covariant derivative $\mathrm{D}_{\mu}$, which is a variant of the Fock-Ivanenko derivative [3], acts as follows:

$$
\mathrm{D}_{\mu} \phi_{A}=\partial_{\mu} \phi_{A}-S_{A}^{\beta} A_{\mu}\left(x^{\mu}\right) \phi_{\beta}
$$

In the complex representation $\phi=\phi_{0}\left(x^{\mu}\right) e^{i \varphi\left(x^{\mu}\right)}$, the covariant derivative takes the form

$$
\mathrm{D}_{\mu} \phi=\partial_{\mu} \phi+i A_{\mu} \phi \text {. }
$$

In field theory, the covariant derivative usually includes the charge $e$ as a characteristic of the interaction with the field, $\mathrm{D}_{\mu} \phi=\partial_{\mu} \phi+i e A_{\mu} \phi$. Here we put $e=1$ for simplicity.

Generalization of external calculus to complex-valued forms implies the replacement $\partial_{\mu} \rightarrow \mathrm{D}_{\mu}$. The consequence of this is the loss of the nilpotency property of the external differential, as occurred above for the covariant differential on Riemannian manifold. Namely, on Minkowski space we have (see [9] for details)

$$
d^{2} \phi=c^{\mathrm{v}} D_{\mathrm{v}}\left(c^{\mu} D_{\mu} \phi\right)=\frac{i}{2} F_{\mu v} \phi c^{\mu} c^{\mathrm{v}},
$$

where $F_{\mu v}=\partial_{\mu} A_{v}-\partial_{v} A_{\mu}$.

Explicitly, taking into account the rule $\partial_{\mu} \rightarrow \mathrm{D}_{\mu}$, the action of the scalar field (8) takes the usual form for complex scalar field interacting with exterior vector field $A^{\mu}$ :

$$
S_{\phi}=\left(\partial_{\mu} \phi^{*}-i A_{\mu} \phi^{*}\right)\left(\partial^{\mu} \phi+i A^{\mu} \phi\right)-m^{2} \phi^{*} \phi .
$$

\section{The Actions of Electromagnetic and Gravitation Fields}

The expression (9) allows us to define the differential of the basis vector in the form 


$$
d e_{A}=e_{B} S_{A}^{\beta} A_{\mu}\left(x^{\mu}\right) c^{\mu} .
$$

For double differential we have

$$
d^{2} e_{A}=e_{B} S_{A}^{\beta} A_{\mu}\left(x^{\mu}\right) c^{\mu}+e_{B} S_{A}^{\beta} \partial_{v} A_{\mu}\left(x^{\mu}\right) c^{v} c^{\mu}=e_{C} S_{\beta}^{C} A_{v} S_{A}^{\beta} A_{\mu}\left(x^{\mu}\right) c^{v} c^{\mu}+e_{B} S_{A}^{\beta} \partial_{v} A_{\mu}\left(x^{\mu}\right) c^{v} c^{\mu}
$$

Obviously, the first term is identically equals to zero. So, we get

$$
d^{2} e_{A}=\frac{1}{2} e_{B} S_{A}^{\beta} F_{\mu v} d x^{\mu} \wedge \mathrm{d} x^{v} .
$$

Taking into account the relation $S_{\beta}^{A^{\dagger}}=-S_{\beta}^{A}$ and (7) we have

$$
\begin{aligned}
\left(d^{2} e^{A}, d^{2} e_{A}\right) & =-\frac{1}{2} \int e^{e} S_{C}^{A} F_{\mu \mathrm{v}} g^{\mu a} g^{\mathrm{v} \beta} e_{D} S_{A}^{D} F_{a \beta} \sqrt{-g} d \Omega=\frac{1}{2} \int e^{A} F_{\mu \mathrm{v}} g^{\mu a} g^{\mathrm{v} \beta} e_{A} F_{a \beta} \sqrt{-g} d \Omega \\
& =\int F_{\mu v} F^{\mu \mathrm{v}} \sqrt{-g} d \Omega .
\end{aligned}
$$

Finally, the action of the electromagnetic field $S_{E M}=-\frac{1}{16 \pi} \int F_{\mu v} F^{\mu v} \sqrt{-g} d \Omega$ can be rewritten in the terms of structure elements of tangent bundle space,

$$
S_{E M}=-\frac{1}{16 \pi}\left(d^{2} e^{A}, d^{2} e_{A}\right) .
$$

Note that the inner square of the first differentials of the basis vectors $\left(d e^{A}, d e_{A}\right)$, by virtue of (10) is proportional to $A_{\mu} A^{\mu}$. Such term is not included in the action of charged particles and the electromagnetic field due to the zero-mass assumption for photons. The action for general relativity case can be constructed almost in the same way, with the basis have to be taken due to (4). For basis fields $c^{a}=h_{\mu}^{a} c^{\mu}$ and $c_{a}=h_{a}^{v} \bar{c}_{v}$.we have

$$
\left(c^{a},(d \hat{\bar{\delta}}+\hat{\bar{\delta}} d) c_{a}\right)=-\int h_{\alpha}^{a} R_{v x} h_{a}^{v} g^{\alpha x} \sqrt{-g} d \Omega=-\int R \sqrt{-g} d \Omega,
$$

where $R=R_{\alpha \beta} g^{\alpha \beta}$ is the scalar curvature.

Thus, the Einstein-Hilbert action [5] for the gravitational field canbe written as

$$
S_{E H}=-\frac{1}{16 \pi G}\left(c^{a},(d \hat{\bar{\delta}}+\hat{\bar{\delta}} d) c_{a}\right) \text {. }
$$

\section{Conclusion}

In the present work, we demonstrated that the action for scalar, electromagnetic, and gravitation fields can be represented as the sum of the internal squares of the scalar field, the basis fields and their differentials,

$$
S=\frac{1}{2}\left(d \phi^{\dagger}, d \phi\right)-\frac{m^{2}}{2}\left(\phi^{\dagger}, \phi\right)-\frac{1}{16 \pi}\left(d^{2} e^{A}, d^{2} e_{A}\right)-\frac{1}{16 \pi G}\left(c^{a},(d \hat{\bar{\delta}}+\hat{\delta} d) c_{a}\right) .
$$

As a final remark we note that construction of a Lagrangian as 4-form on Riemannian space imposes some restrictions on the acceptable forms of action of field theoretical models. 


\section{REFERENCES}

1 Hehl F.W. and Obukhov Y.N. E'lie Cartan's torsion in geometry and in field theory, an essay. arXiv:0711.1535v1 [gr-qc] 9 Nov2007.

2 Trautman A. Einstein-Cartan Theory. arXiv:gr-qc/0606062v1 14 Jun 2006

3 Aldrovandi R. and Pereira J.G. Teleparallel Gravity: An Introduction. New York, London: Springer Dordrecht Heidelberg, 2013, 202p.

4 Eliashberg Y., Givental A. and Hofer H. Introduction to Symplectic Field Theory. Basel: Birkhauser Verlag, 2000, 102p.

5 Clifton T., Ferreira P.G., Padilla A. and Skordis C. Modified Gravity and Cosmology. Physics Reports, 2012, No 513, pp.1-189.

6 Utiyama R. Invariant theoretical interpretation of interaction. Phys. Rev., 1956, Vol. 101, No 5, pp.1597-1608.

7 Aringazin A.K., Arkhipov V.V. and Kudusov A.S. BRST Approach to Hamiltonian systems // arXiv:hepth/9811026

8 Atiah M.F. and Singer I.M. The index of elliptic operators I. Uspekhi Mat. Nauk, 1968, Vol. 23, No 5(143), pp.99-142.

9 Arkhipov V.V. Minimal Cohomological Model of a Scalar Field on a Riemannian Manifold. Russ. Phys. J., 2018., Vol. 60, No 12, pp.2051-2063. 